Conclusion: Target organ associations could support distinctive subphenotypes in PAN. Factor 1 seems the most severe form. Patients with FMF or DADA2 have distinct target organ associations. The jury is out to decide whether these patients should be classified as 'vasculitis associated with probable etiology' just as HBV-related-PAN. Factor 4 might define a different subphenotype (ANCAmedium vessel vasculitis?)

Disclosure of Interests: Omer Karadag: None declared, Ertugrul Cagri Bolek: None declared, Shunsuke Furuta: None declared, Giacomo Emmi: None declared, ALOJZIJA HOCEVAR: None declared, Andrea Hinojosa-Azaola: None declared, Aladdin J Mohammad Speakers bureau: lecture fees from Roche and Elli Lilly Sweden, PI (GiACTA study), Serdal Ugurlu: None declared, Fatma Alibaz-Oner: None declared, Ayten Yazici: None declared, Luca Quartuccio: None declared, Enrica Bozzolo: None declared, Lorenzo Dagna Grant/research support from: Abbvie, BMS, Celgene, Janssen, MSD, Mundipharma Pharmaceuticals, Novartis, Pfizer, Roche, SG, SOBI, Consultant of: Abbvie, Amgen, Biogen, BMS, Celltrion, Novartis, Pfizer, Roche, SG, and SOBI, Giuseppe Alvise Ramirez: None declared, Luca Cantarini: None declared, Gina Gregorini: None declared, Jeannin Guido: None declared, Sara Monti: None declared, Eduardo Martin-Nares: None declared, Franco Schiavon: None declared, Roberto Padoan: None declared, Hajime Kono: None declared, Augusto Vaglio: None declared, Saadettin Kılıçkap: None declared, Ali Insan Ertenli: None declared, Haner Direskeneli: None declared, Seza Özen Consultant of: Novartis, Pfizer, Speakers bureau: SOBI, Novartis, David Jayne Grant/research support from: ChemoCentryx, GSK, Roche/Genentech, Sanofi-Genzyme, Consultant of: Astra-Zeneca, ChemoCentryx, GSK, InflaRx, Takeda, Insmed, Chugai, Boehringer-Ingelheim

DOI: 10.1136/annrheumdis-2020-eular.4635

\section{SAT0244 PROGNOSIS OF LARGE VESSEL INVOLVEMENT IN LARGE VESSEL VASCULITIS}

M. Vautier ${ }^{1}$, A. Dupont ${ }^{2}$, H. De Boysson ${ }^{3}$, C. Comarmond ${ }^{1}$, T. Mirault ${ }^{4}$, A. Mekinian ${ }^{5}$, M. Lambert ${ }^{6}$, Y. Ferfar ${ }^{1}$, A. Aouba ${ }^{3}$, P. Cacoub ${ }^{1}$, M. Resche-Rigon ${ }^{2}$, D. Saadoun'. ' University Hospitals Pitié Salpêtrière - Charles Foix, Paris, France: ${ }^{2}$ Hospital _ Saint-Louis Ap-Hp, Paris, France; ${ }^{3}$ Caen, Caen, France; ${ }^{4}$ Hôpital Européen Georges-Pompidou, Paris, France; ${ }^{5}$ Hospital Saint-Antoine Ap-Hp, Paris, France; ${ }^{6}$ Lille, Lille, France

Background: Giant cell arteritis (GCA) and Takayasu arteritis (TAK) are the two main forms of large vessels vasculitis (LVV). Vessel inflammation leads to aneurysms, wall thickening, stenosis, and, in some cases, complete occlusion of the artery. Due to the wide variation in the course of LVV, predicting outcome is challenging. To our knowledge, data regarding prognosis factors of LVI in LVV patients and comparison of outcome of LVI in GCA and TAK are lacking. An early identification of patients with higher mortality could help to prevent deaths and vascular complications.

Objectives: To assess prognosis factors and outcome of large vessel involvement (LVI) in large vessels vasculitis (LVV) patients.

Methods: Retrospective multicenter study of characteristics and outcomes of 417 patients with LVI including 299 Takayasu arteritis (TAK) and 118 Giant cell arteritis (GCA-LVI) were analyzed. Logistic regression analysis assessed prognosis factors in LVV patients. Outcome of LVI among TAK and GCA-LVI patients (ischemic complications, aneurysms complications, relapses and revascularization) were assessed.

Results: In multivariable analysis, stroke/transient ischemic attack [HR: 3.63 (1.46 - 9.04), $p=0.006$ ] was independently associated with vascular complications in LVV. The 10-years aneurysm free survival was significantly lower [67\% $(48$ - 93) vs $89 \%(84-95), p=0.02]$ in GCA-LVI compare to TAK patients. The 5-years relapse free survival was significantly lower $[47 \%$ $(37-60)$ vs $69 \%(63-75), p<0.001$,$] in GCA-LVI compare to TAK patients.$ The 10 -years revascularization free survival was significantly lower [55\% $(48-64)$ vs $76 \%(59-99), p<0.001]$ in TAK compare to GCA-LVI patients. After a median follow-up of 5 years, 16 (5.4\%) TAK and 7 (5.9\%) GCALVI patients died, mainly of aneurysm (26\%) and ischemic complications $(26 \%)$.

Conclusion: This large nationwide cohort of LVI provided prognosis factors of vascular complications in LVV patients. TAK and GCA-LVI have different long-term outcome in term of aneurysm development, relapse and revascularization.

Disclosure of Interests: None declared

DOI: 10.1136/annrheumdis-2020-eular.5252

\section{SAT0245 RENAL INVOLVEMENT AT ONSET IN ANTI- NEUTROPHIL CYTOPLASMIC ANTIBODY (ANCA)- ASSOCIATED VASCULITIS: A MAJOR INDEPENDENT RISK FACTOR FOR RENAL RELAPSE}

M. Felicetti ${ }^{1}$, A. Ortolan ${ }^{1}$, A. C. Frigo ${ }^{2}$, R. Padoan ${ }^{1}$, M. Gasparotto ${ }^{1}$, M. Lorenzin ${ }^{1}$, A. Doria ${ }^{1}$, R. Ramonda ${ }^{1}$, F. Schiavon ${ }^{1} .{ }^{1}$ University of Padova, Department of Medicine DIMED-Rheumatology Unit, Padova, Italy; ${ }^{2}$ University of Padova, Department of Cardiac, Thoracic and Vascular Sciences-Biostatistics, Epidemiology and Public Health Unit, Padova, Italy

Background: In ANCA-associated vasculitis (AAV), renal relapses are cause for concern as they are unpredictable and predictors of end-stage renal disease (ESRD).

Objectives: We aimed to assess the frequency of major renal (MR) relapses in AAV in our cohort and identify independent predictors of the first MR relapse at diagnosis.

Methods: We performed a retrospective monocentric observational study in our Vasculitis clinic from January 2000 to August 2019. Inclusion criteria were: 1) granulomatosis with polyangiitis (GPA), microscopic polyangiitis (MPA) and limited kidney disease (LKD) diagnosis fulfilling EMA algorithm criteria; 2 ) achievement of a stable remission, defined as absence of vasculitis symptoms or signs and adherence to the prednisone taper during remission-induction treatment. We excluded patients who developed ESRD before remission and those with incomplete data during the follow-up. Major renal (MR) relapses were defined as occurrence of at least one major item of renal Birmingham Vasculitis Activity Score version 3 (BVASv3).

All remitted patients were allocated in two subgroups: patients without MR relapse and patients with MR relapse. Univariate and multivariable analysis of first MR relapse predictors was performed with Fine and Gray (F\&G) sub distribution hazards model to assess all competitive risks (progression to ESRD without MR relapse and death before MR relapse). Due to the relatively low frequency of events and the risk of overfitting, we performed several multivariable models with three variables, as recommended by Peduzzi e $\mathrm{al}^{1}$. The best multivariable model was selected accordingly to the Akaike information criterion (AIC).

Results: 96 (53\% females) patients met the inclusion criteria: 74 GPA, 21 MPA and 1 LKD. Median age at diagnosis was 54 (44-64) years. ANCA testing was present in 94 patients, 85 were ANCA positive: 56 c-ANCA/PR3, 28 p-ANCA/MPO and 1 double positivity.

During a median follow-up (FU) of 54.5 months (29.3-96.5), we observed 19 MR relapses in 17 patients while 2 patients progressed to ESRD, 3 died without events and 76 reported no MR relapse. Density-incidence of MR relapses since remission was 3.6/100 person-year (Cl 95\% 2.2-5.6). Median time to first MR relapse after remission was 33 months (14-67.5)

At first MR relapse, 8 (53.3\%) patients were on steroids while $10(66.7 \%)$ were on immunosuppressant (5 azathioprine, 5 mycophenolate). In 2 cases, data about remission-maintenance treatment was not available.

MR relapses were observed only in ANCA positive patients with a significantly higher frequency of skin, kidney and nerve involvement at diagnosis $(41.2 \%$ vs $17.7 \%, p=0.034,94.1 \%$ vs $57.0 \% p=0.004$, and $52.9 \%$ vs $25.3 \% p=0.024$, respectively); while Ear, Nose and Throat (ENT) involvement was significantly lower (35.3\% vs $62.0 \% \mathrm{p}=0.043$ ). Mean BVASv3 at diagnosis scored significantly higher in MR relapse group (24.1 \pm 6.2 vs $18.1 \pm 8.1 . p=0.007)$.

At multivariable analysis with $F \& G$ model, renal involvement and induction treatment without cyclophosphamide and/or Rituximab at diagnosis were independent predictors of MR relapse (sHR 20.4 (2.6-158.2), $\mathrm{p}=0.004$ and sHR 4.2 (1.5-12.0), $\mathrm{p}=0.007$, respectively). Moreover, there was a trend of higher risk of MR relapse in PR3-ANCA (sHR 2.5 (0.9-7.1), $p=0.091$ ).

Conclusion: Renal involvement at diagnosis and milder remission-induction treatment regimens resulted in a significantly higher risk of MR relapse during the FU in our cohort. PR3-ANCA specificity was not an independent predictor of MR relapse, even if we observed a trend of higher MR relapse risk with this covariate.

\section{References:}

[1] Peduzzi P et al. A simulation study of the number of events per variable in logistic regression analysis. J Clin Epidemiol. 1996;49(12):1373-9.

Disclosure of Interests: Mara Felicetti: None declared, Augusta Ortolan: None declared, Anna Chiara Frigo: None declared, Roberto Padoan: None declared, Michela Gasparotto: None declared, Mariagrazia Lorenzin: None declared, Andrea Doria Consultant of: GSK, Pfizer, Abbvie, Novartis, Ely Lilly, Speakers bureau: UCB pharma, GSK, Pfizer, Janssen, Abbvie, Novartis, Ely Lilly, BMS, Roberta Ramonda Speakers bureau: Novartis, Celgene, Janssen, Pfizer, Abbvie, Lilly, Franco Schiavon: None declared

DOI: 10.1136/annrheumdis-2020-eular.5830 